\title{
Projeção da distribuição diamétrica de povoamentos de eucalipto em diferentes amplitudes de classe
}

\author{
Carlos Alberto Araújo Júnior(1), Gilciano Saraiva Nogueira(2), Marcio Leles Romarco de Oliveira(2), \\ Rodrigo Otávio Veiga de Miranda ${ }^{(3)}$, Renato Vinícius Oliveira Castro(1) e Eduardo Pelli(2)
}

\begin{abstract}
(1)Universidade Federal de Viçosa, Departamento de Engenharia Florestal, CEP 36570-000 Viçosa, MG. E-mail: araujocaj@gmail.com, castrorvo@ymail.com (2)Universidade Federal dos Vales do Jequitinhonha e Mucuri, Departamento de Engenharia Florestal, Rua da Glória, no 187, CEP 39100-000 Diamantina, MG. E-mail: nogueirags@yahoo.com.br, marcioromarco@gmail.com, eduardopell@gmail.com (3)Universidade Estadual do Oeste do Paraná, Departamento de Engenharia Florestal, PR 153, Km 07, CEP 84.500-000 Irati, PR. E-mail: rov_miranda@yahoo.com.br.
\end{abstract}

Resumo - O objetivo deste trabalho foi verificar a qualidade da modelagem da distribuição diamétrica de povoamentos equiâneos de eucalipto, de acordo com a variação na amplitude de classe utilizada. Avaliaram-se tanto o ajuste da função aos dados observados quanto a projeção dos parâmetros da função, por meio de um modelo de distribuição diamétrica. Foram utilizados dados de 48 parcelas permanentes, obtidos em quatro ocasiões. Constatou-se equivalência estatística entre as distribuições observadas e as estimadas pela função Weibull, em todas as situações. A função Weibull ajustou-se adequadamente aos dados, independentemente da amplitude de classe; no entanto, a redistribuição teórica dos diâmetros por classe, por meio de um modelo de distribuição diamétrica, foi afetada pela amplitude de classe.

Termos para indexação: Eucalyptus grandis x Eucalyptus urophylla, amplitude de classe, densidade de probabilidade, distribuição diamétrica, função Weibull, máxima verossimilhança.

\section{Projection of diameter distribution of eucalyptus stands considering different class ranges}

\begin{abstract}
The objective of this work was to assess the fitting quality of the diameter distribution modeling of an even-aged stand of eucalyptus, according to the variation in the diameter class range. Both the function fitting to the observed data and the projection of the function parameters were evaluated, using a diametric distribution model. Data from 48 permanent plots, obtained at four occasions, was used. Statistical equivalence between the observed distributions and the estimated ones by the Weibull function were evidenced in all situations. The Weibull function fitted properly to the observed data independently of class range; however, the theoretical redistribution of diameter per class, using a diametric distribution model, was affected by class range.
\end{abstract}

Index terms: Eucalyptus grandis $\mathrm{x}$ Eucalyptus urophylla, class range, probability density, diametric distribution, Weibull function, maximum likelihood.

\section{Introdução}

A caracterização da distribuição diamétrica pode indicar o potencial de utilização de povoamentos florestais, principalmente quando se trabalha com multiprodutos. A distribuição diamétrica de determinado povoamento ou floresta é facilmente avaliada por meio da medição direta das árvores, com os diâmetros agrupados em classes de amplitudes definidas.

No entanto, somente o conhecimento acerca da distribuição diamétrica atual de um povoamento nem sempre é suficiente. Quando se deseja analisar o crescimento e a produção por classe de diâmetro, é necessário empregar modelos de distribuição diamétrica. Esses modelos são baseados em funções de densidade de probabilidade, das quais destaca-se a função Weibull, por sua relativa simplicidade e flexibilidade em representar tendências (Bailey \& Dell, 1973). A função Weibull ainda tem a vantagem de seus parâmetros serem facilmente correlacionados com variáveis do povoamento, o que possibilita que sejam calculados para uma idade futura, e permite estudos sobre crescimento e produção das florestas equiâneas (Campos \& Leite, 2009), conforme verificado nos trabalhos de Mabvurira et al. (2002), 
Cao (2004) e Eisfeld et al. (2005). Ao comparar a função Weibull a outras funções de densidade de probabilidade, Abreu et al. (2002), Catalunha et al. (2002) e Arce (2004) constataram a superioridade da função Weibull.

O ajuste de uma função probabilística pode ser realizado por diferentes métodos, dos quais se destacam o dos percentis, o dos momentos e o de máxima verossimilhança (Arce, 2004). O uso de um ou outro método irá depender da precisão requerida e da função utilizada. O método de máxima verossimilhança é o mais indicado para o ajuste da função Weibull, já que os procedimentos computacionais otimizam o processo de estimativa dos parâmetros.

A amplitude de classe de diâmetro para estudos de crescimento e produção tem sido definida de acordo com o porte das árvores. Assim, encontramse amplitudes de 1 a $5 \mathrm{~cm}$, para florestas de eucalipto e de teca (Scolforo \& Thierschi, 1998; Soares, 2006; Campos \& Leite, 2009), e amplitudes acima disso para florestas inequiâneas (Umaña \& Alencar, 1998; Cunha et al., 2002). No entanto, a definição dessa amplitude pode não considerar a mudança do porte das árvores ao longo do tempo, o que torna importantes os estudos direcionados para verificar as inconsistências na projeção da distribuição diamétrica ocasionadas por essa variação.

O objetivo deste trabalho foi verificar a qualidade do ajuste da função Weibull aos dados de distribuição diamétrica de povoamentos equiâneos de eucalipto, de acordo com a variação na amplitude de classe utilizada.

\section{Material e Métodos}

Os dados utilizados foram obtidos de povoamentos do híbrido Eucalyptus grandis x Eucalyptus urophylla, da empresa Copener Florestal, na região nordeste do Estado da Bahia. Foram medidas 48 parcelas permanentes, cada uma com área útil de aproximadamente $2.600 \mathrm{~m}^{2}$, nas idades de 27, 40, 50 e 58 meses. Em todas as medições, foram registradas, nas parcelas, a altura total das 15 primeiras árvores (ht), altura de 5 árvores dominantes (hd) e o diâmetro a 1,3 m de altura (DAP) de todas as árvores.

Para avaliar a influência da amplitude de classe na modelagem da distribuição diamétrica, os diâmetros foram agrupados em classes de cinco diferentes amplitudes, escolhidas arbitrariamente: 1, 2, 3, 4 e $5 \mathrm{~cm}$.

A função densidade de probabilidade de Weibull, com dois parâmetros, foi ajustada por parcela, em todas as idades e amplitudes de classe consideradas, pela equação:

$f(x)=\left(\frac{\gamma}{\beta}\right)\left(\frac{x}{\beta}\right)^{(\gamma-1)} e^{-\left(\frac{x}{\beta}\right)^{\gamma}}$

em que: $x$ é o centro da classe diamétrica, em cm; $\gamma$ é o parâmetro de forma; $\beta$ é o parâmetro de escala; e e é a função exponencial.

O método empregado para a estimação dos parâmetros da função Weibull foi o da máxima verossimilhança, conforme definido por Gove \& Fairweather (1989). Inicialmente, a função de verossimilhança da equação 1 foi obtida pelo produtório $\left[\prod_{i-1}^{n} f\left(x_{i}\right)\right]$ tendo-se aplicado, em seguida, o logaritmo para obtenção da função log-verossimilhança. A derivada da função log-verossimilhança, em relação a cada um dos parâmetros, e sua igualdade a zero, permitiu obter o seguinte sistema de equações:

$$
\begin{aligned}
& \hat{\beta}=\left(\frac{\sum_{\mathrm{i}=1}^{\mathrm{n}} \mathrm{xi}^{\hat{\gamma}}}{\mathrm{n}}\right)^{\frac{1}{\hat{\gamma}}} \\
& \frac{\sum_{\mathrm{i}=1}^{\mathrm{n}} \mathrm{xi}^{\gamma} \ln \mathrm{xi}}{\sum_{\mathrm{i}=1}^{\mathrm{n}} \mathrm{xi}^{\gamma}}-\frac{\sum_{\mathrm{i}=1}^{n} \ln \mathrm{xi}}{\mathrm{n}}-\frac{1}{\gamma}=0
\end{aligned}
$$

em que: $\mathrm{n}$ é o número de árvores por parcela; e x, $\gamma$ e $\beta$ conforme definidos anteriormente.

O sistema das equações que compõem o modelo de distribuição diamétrica, apresentado por Nogueira et al. (2005), foi ajustado, tendo-se relacionado os parâmetros da função Weibull aos atributos do povoamento. Assim, a partir das distribuições diamétricas observadas na idade de 27 meses, foram prognosticadas distribuições quanto às idades de 40, 50 e 58 meses, por meio do modelo de distribuição diamétrica ajustado, comparadas às distribuições observadas em cada amplitude. Esse procedimento foi semelhante ao de Leite et al. (2006), em que os autores 
realizaram a predição dos parâmetros da função Weibull com base nos parâmetros observados em uma idade.

O sistema de equações foi definido por:

$$
\begin{aligned}
& \mathrm{D}_{\text {mín } 2}=\mathrm{D}_{\min 1} * \mathrm{e}^{-\mathrm{c}_{0} *\left(\mathrm{I}_{2}^{\mathrm{c}_{1}}-\mathrm{I}_{1}^{\mathrm{c}}\right)} * \varepsilon \\
& \mathrm{D}_{\text {máx } 2}=\mathrm{D}_{\text {máx } 1} *\left(\mathrm{I}_{1} / \mathrm{I}_{2}\right)+\mathrm{c}_{2}\left(1-\mathrm{I}_{1} / \mathrm{I}_{2}\right) * \beta_{1}+\varepsilon \\
& \operatorname{Ln} \gamma_{2}=\operatorname{Ln} \gamma_{1} * \mathrm{e}^{-\mathrm{c}_{3} *\left(\mathrm{I}_{2}^{\mathrm{c} 4}-\mathrm{I}_{1}^{\mathrm{c} 4}\right)} * \varepsilon \\
& \beta_{2}=\beta_{1} *\left(I_{1} / I_{2}\right)+c_{5}\left(1-I_{1} / I_{2}\right) * D_{\text {máx } 2}+\varepsilon \\
& \mathrm{N}_{2}=\mathrm{N}_{1} * \mathrm{e}^{-\mathrm{c}_{6} *\left(\mathrm{I}_{2}^{\mathrm{c} 7}-\mathrm{I}_{1}^{\mathrm{c} 7}\right)} * \varepsilon \\
& \mathrm{Ht}=\mathrm{c}_{8}+\mathrm{c}_{9} \mathrm{I}+\mathrm{c}_{10} \mathrm{~S}+\mathrm{c}_{11} \operatorname{Ln}\left(\mathrm{I}^{*} \text { dap }\right)+\varepsilon \\
& \operatorname{Ln}(\mathrm{V})=\mathrm{c}_{12}+\mathrm{c}_{13} * \operatorname{Ln}(\text { dap })+\mathrm{c}_{14} * \operatorname{Ln}(\mathrm{Ht})+\varepsilon
\end{aligned}
$$

em que: $I_{1}$ e $I_{2}$ são as idades inicial e final, respectivamente; $d_{\text {mín1 }}$ e $d_{\text {mín2 }}$ são os diâmetros mínimos observados por parcela, nas idades inicial e final; $d_{\text {máx } 1} e$ $\mathrm{d}_{\text {máx } 2}$ são os diâmetros máximos observados por parcela, nas idades inicial e final; $\mathrm{N}_{1}$ e $\mathrm{N}_{2}$ são as frequências totais observadas por parcela, nas idades inicial e final; $\gamma_{1}, \beta_{1}, \gamma_{2}$ e $\beta_{2}$ são os parâmetros da função Weibull, nas idades inicial e final; $\mathrm{S}$ é o índice de local; ht é a altura da árvore; dap é o diâmetro a 1,30 m do solo; V é o volume individual da árvore; $\mathrm{c}_{0} \mathrm{a} \mathrm{c}_{14}$ são os parâmetros a serem estimados; $\varepsilon$ é o erro aleatório; e os demais termos conforme definidos anteriormente.

As distribuições estimadas pela função Weibull e as prognosticadas pelo modelo de distribuição diamétrica foram comparadas pelo teste não paramétrico de Kolmogorov-Smirnov, a 5\% de probabilidade:

$\mathrm{D}=\operatorname{máx}|\mathrm{Fo}(\mathrm{x})-\mathrm{Fe}(\mathrm{x})|$

em que: $\mathrm{D}$ é a diferença máxima absoluta; $\mathrm{F}_{\mathrm{o}}(\mathrm{x})$ e $\mathrm{F}_{\mathrm{e}}(\mathrm{x})$ são as frequências acumuladas observadas e esperadas, respectivamente.

Para identificar possíveis tendenciosidades nas estimativas de produção volumétrica total e por classe de diâmetro, foram aplicadas as estatísticas utilizadas por Murphy \& Sternitzke (1979):
$\mathrm{RQEM}=\sqrt{\frac{1}{\mathrm{~N}} \sum_{\mathrm{i}=1}^{\mathrm{N}}\left(\hat{\mathrm{V}}_{\mathrm{i}}-\mathrm{V}_{\mathrm{i}}\right)^{2}}$

$\mathrm{MD}=\frac{1}{\mathrm{~N}} \sum_{\mathrm{i}=1}^{\mathrm{N}}\left(\hat{\mathrm{V}}_{\mathrm{i}}-\mathrm{V}_{\mathrm{i}}\right)^{2}$

$\operatorname{MDP}=\frac{1}{\mathrm{~N}} \sum_{\mathrm{i}=1}^{\mathrm{N}}\left(\frac{\hat{\mathrm{V}}_{\mathrm{i}}-\mathrm{V}_{\mathrm{i}}}{\mathrm{V}_{\mathrm{i}}}\right) * 100$

em que: RQEM é a raiz quadrada do erro médio; MD é a média das diferenças; MDP é a média das diferenças percentuais; $\mathrm{N}$ é o número de casos, ou seja, o número de parcelas com volume observado nas classes de diâmetros indicadas; e $\hat{V}_{i}$ e $V_{i}$ são os volumes estimados e observados para cada classe de diâmetro, respectivamente.

\section{Resultados e Discussão}

As distribuições diamétricas observadas apresentaram tendência normal até a amplitude de classe de $3,0 \mathrm{~cm}$, para todas as idades consideradas. Para algumas parcelas com amplitude acima de $4,0 \mathrm{~cm}$, esta tendência não foi observada, em razão de a quantidade de classes ter sido menor que três. À medida que a amplitude de classe aumentou, a quantidade de classes diminuiu e passou de 7 a 9 classes (1,0 cm de amplitude) para apenas 2 classes (5,0 $\mathrm{cm}$ de amplitude) (Tabela 1).

Apesar da variação existente no número de classes, a função Weibull se ajustou adequadamente aos dados observados (Figura 1), o que comprova sua flexibilidade em descrever tendências diversas, conforme mencionado por Nogueira (2003),

Tabela 1. Frequência de parcelas em função da quantidade de classes observadas na distribuição diamétrica, em diferentes amplitudes, para a idade de 27 meses.

\begin{tabular}{lccccc}
\hline Quantidade de classes & \multicolumn{5}{c}{ Amplitude de classe (cm) } \\
\cline { 2 - 6 } & 1,0 & 2,0 & 3,0 & 4,0 & 5,0 \\
\hline 2 & - & - & - & 26 & 48 \\
3 & - & - & 26 & 22 & - \\
4 & - & 26 & 22 & - & - \\
5 & - & 22 & - & - & - \\
6 & 02 & - & - & - & - \\
7 & 24 & - & - & - & - \\
8 & 20 & - & - & - & - \\
9 & 02 & - & - & - & - \\
\hline
\end{tabular}


Leite et al. (2006) e Soares (2006). À medida que as árvores cresceram, a distribuição se deslocou para a direita, e a função Weibull conseguiu captar esse deslocamento da distribuição (Figura 1).

O teste de Kolmogorov-Smirnov não foi significativo para todas as parcelas e em todas as idades analisadas, o que mostra a adequação do ajuste da função aos dados observados.

Assim, a função Weibull mostrou-se flexível o suficiente para descrever tendências que, para este caso, variaram de linear (para as parcelas que tiveram distribuição com apenas duas classes diamétricas) a normal. A qualidade do ajuste pode ser atribuída ao método de máxima verossimilhança para estimação dos parâmetros, conformeNávar-Cháidez\& Contreras-Aviña (2000) e Ayala \& Návar-Cháidez (2002).

Nas Tabelas 2 e 3 encontram-se os coeficientes do modelo de distribuição diamétrica (equações 4 a 8), ajustado com base nos resultados da função de densidade probabilística. Na Tabela 3, encontram-
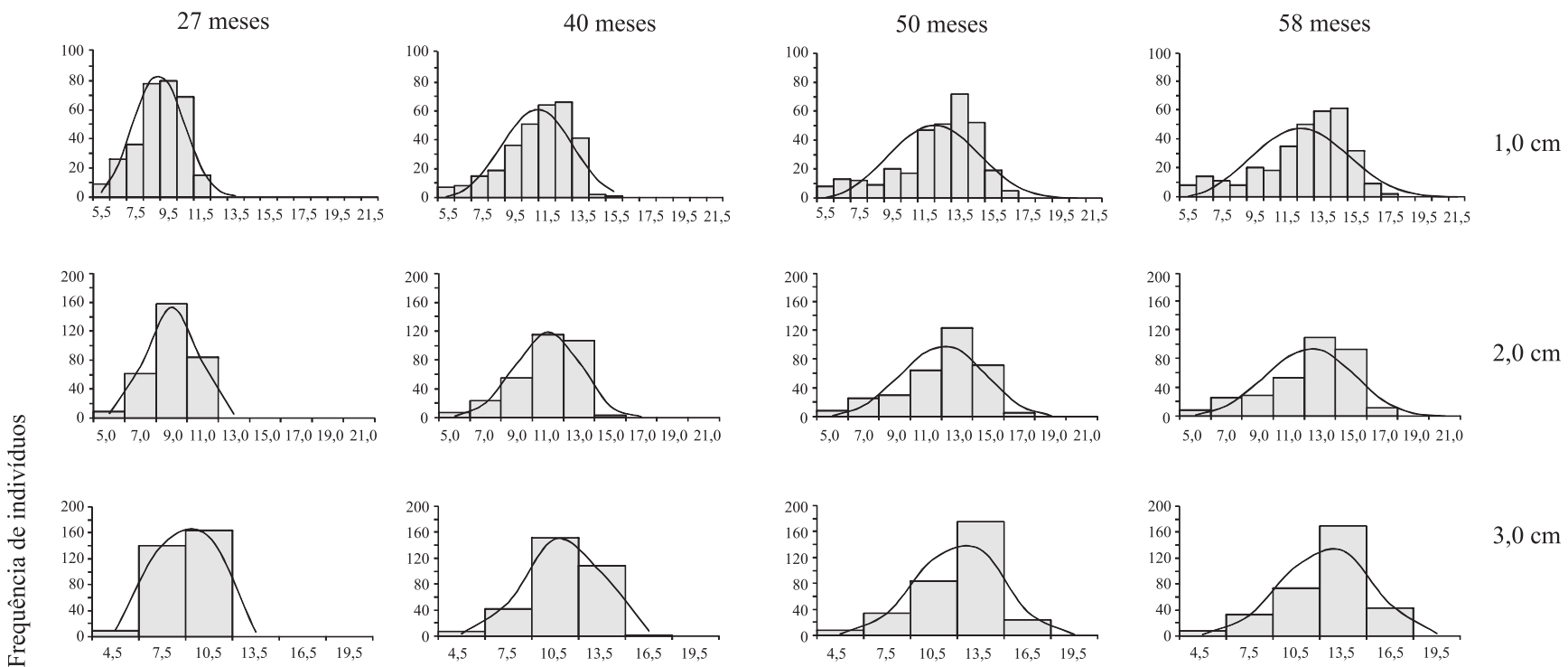

$2,0 \mathrm{~cm}$
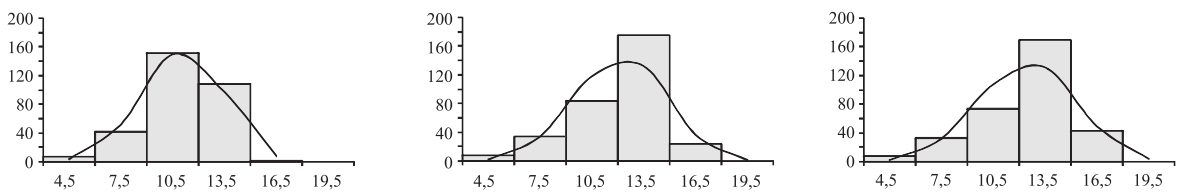

$3,0 \mathrm{~cm}$
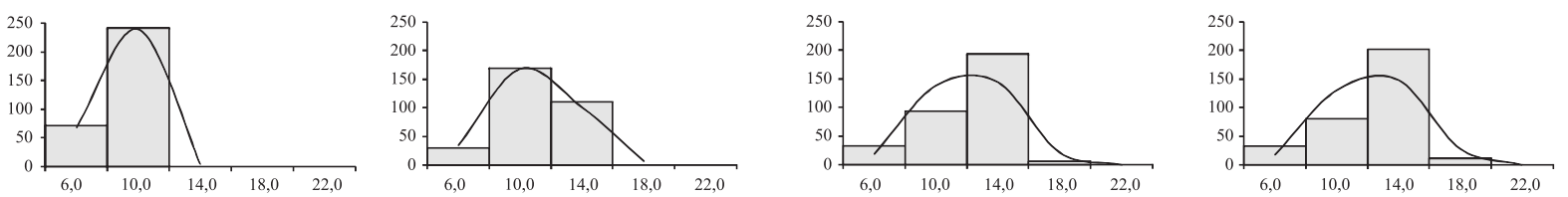

$4,0 \mathrm{~cm}$
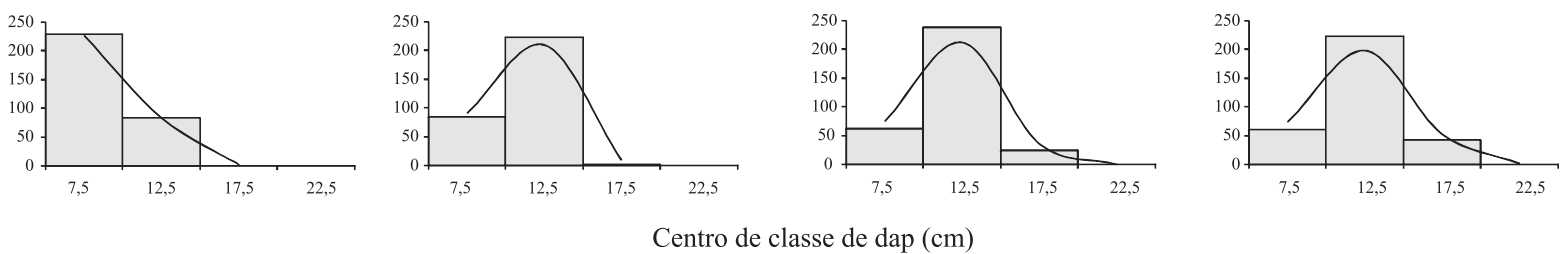

$5,0 \mathrm{~cm}$

Figura 1. Distribuição diamétrica observada (barras) e estimada (linha) para a parcela 48, em cada idade, e para cada amplitude de classe considerada $(1,0,2,0,3,0,4,0$ e 5,0 cm). $\mathrm{O}$ alinhamento vertical representa as idades, e o alinhamento horizontal as amplitudes de classe consideradas.

Tabela 2. Parâmetros estimados para o modelo de distribuição diamétrica, em cada amplitude de classe diamétrica considerada, com os respectivos coeficientes de correlação (r).

\begin{tabular}{|c|c|c|c|c|c|c|c|c|c|c|c|}
\hline \multirow[t]{2}{*}{ Variável dependente } & \multirow[t]{2}{*}{ Parâmetros } & \multicolumn{2}{|c|}{1,0} & \multicolumn{2}{|c|}{2,0} & \multicolumn{2}{|c|}{3,0} & \multicolumn{2}{|c|}{4,0} & \multicolumn{2}{|c|}{5,0} \\
\hline & & Valor & $\mathrm{r}$ & Valor & $\mathrm{r}$ & Valor & $\mathrm{r}$ & Valor & $\mathrm{r}$ & Valor & $\mathrm{r}$ \\
\hline $\operatorname{Ln} \gamma_{2}$ & $\begin{array}{l}\text { c3 } \\
\text { c4 }\end{array}$ & $\begin{array}{c}0,3548 \\
-0,1005\end{array}$ & 0,8957 & $\begin{array}{c}3,2798 \\
-1,1632\end{array}$ & 0,8554 & $\begin{array}{l}0,1759 \\
0,1108\end{array}$ & 0,8500 & $\begin{array}{l}1,2707 \\
0,0849\end{array}$ & 0,5169 & $\begin{array}{r}-1,3379 \\
0,0866\end{array}$ & 0,6818 \\
\hline$\beta_{2}$ & $\mathrm{c} 5$ & 0,7122 & 0,8986 & 0,7789 & 0,9017 & 0,8455 & 0,9005 & 0,784 & 0,8692 & 0,721 & 0,7891 \\
\hline
\end{tabular}


se os coeficientes das equações para estimativa dos atributos diretamente relacionados ao povoamento, ao número de árvores por hectare e aos diâmetros mínimo e máximo, os quais não variaram em função da amplitude.

Os resíduos das estimativas dos parâmetros $\beta$ e $\gamma$, apresentados na Figura 2, mostraram que, independentemente da amplitude de classe, as estimativas de $\beta$ não foram tendenciosas. $\mathrm{O}$ aumento dos valores do parâmetro $\beta$ decorreu do fato de que ele está relacionado com o ponto de inflexão da distribuição. Assim, à medida que a função deslocouse para a direita, tal parâmetro tendeu a aumentar. Para as amplitudes de 4,0 e 5,0 cm, essa tendência tornou-se inconsistente, principalmente pelo fato de a função assumir uma forma linear em grande parte das distribuições analisadas.

$\mathrm{Na}$ análise dos gráficos de resíduos para o parâmetro $\gamma$, observou-se que houve maior dispersão dos erros nas maiores amplitudes de classe $(4,0$ e $5,0 \mathrm{~cm}$ ), o que indica menor precisão das estimativas. Isso ocorreu porque esse parâmetro descreve a forma da distribuição estimada e, para tais amplitudes, houve distribuições com apenas duas classes, o que indica uma distribuição de forma linear, em contraste com o observado em parcelas com mais de três classes.

Com relação à prognose da distribuição diamétrica, o teste de Kolmogorov-Smirnov mostrou-se não significativo $(p<0,05)$ em todas as situações analisadas, o que permitiu aceitar a hipótese de que existe concordância entre as distribuições prognosticadas e observadas.

Tabela 3. Parâmetros estimados para o modelo de distribuição diamétrica em todas as amplitudes, com os respectivos coeficientes de correlação $\left(\mathrm{r}_{\mathrm{yy}}\right)$.

\begin{tabular}{lcrc}
\hline Variável dependente & Parâmetros & \multicolumn{1}{c}{ Valor } & $\mathrm{r}_{\hat{y y}}$ \\
\hline $\mathrm{D}_{\text {min2 }}$ & $\mathrm{c}_{0}$ & $-0,6650$ & \multirow{2}{*}{0,5162} \\
\hline $\mathrm{D}_{\text {máx }}$ & $\mathrm{c}_{1}$ & 0,0072 & \\
\hline \multirow{2}{*}{$\mathrm{N}_{2}$} & $\mathrm{c}_{2}$ & 3,9211 & 0,7522 \\
\hline \multirow{3}{*}{$\mathrm{Ht}$} & $\mathrm{c}_{6}$ & 0,1379 & \multirow{2}{*}{0,9963} \\
& $\mathrm{c}_{7}$ & 0,0104 & \\
\hline & $\mathrm{c}_{8}$ & $-50,0827$ & \\
& $\mathrm{c}_{9}$ & $-0,0530$ & \\
& $\mathrm{c}_{10}$ & 0,7573 & 0,9720 \\
Ln (V) & $\mathrm{c}_{11}$ & 8,5148 & \\
& $\mathrm{c}_{12}$ & $-10,0114$ & \\
& $\mathrm{c}_{13}$ & 1,7324 & 0,9990 \\
& $\mathrm{c}_{14}$ & 1,1538 & \\
\hline
\end{tabular}

Na Tabela 4, os resultados das estatísticas de MD, MDP e RQEM indicaram diferença entre valores estimados e observados, e entre valores prognosticados pelo modelo de distribuição diamétrica e os observados para a produção volumétrica $\left(\mathrm{m}^{3} \mathrm{ha}^{-1}\right)$, em todas as classes de diâmetro e amplitudes de classe da distribuição. Na comparação dos valores de MD, MDP e RQEM, associados às distribuições estimadas
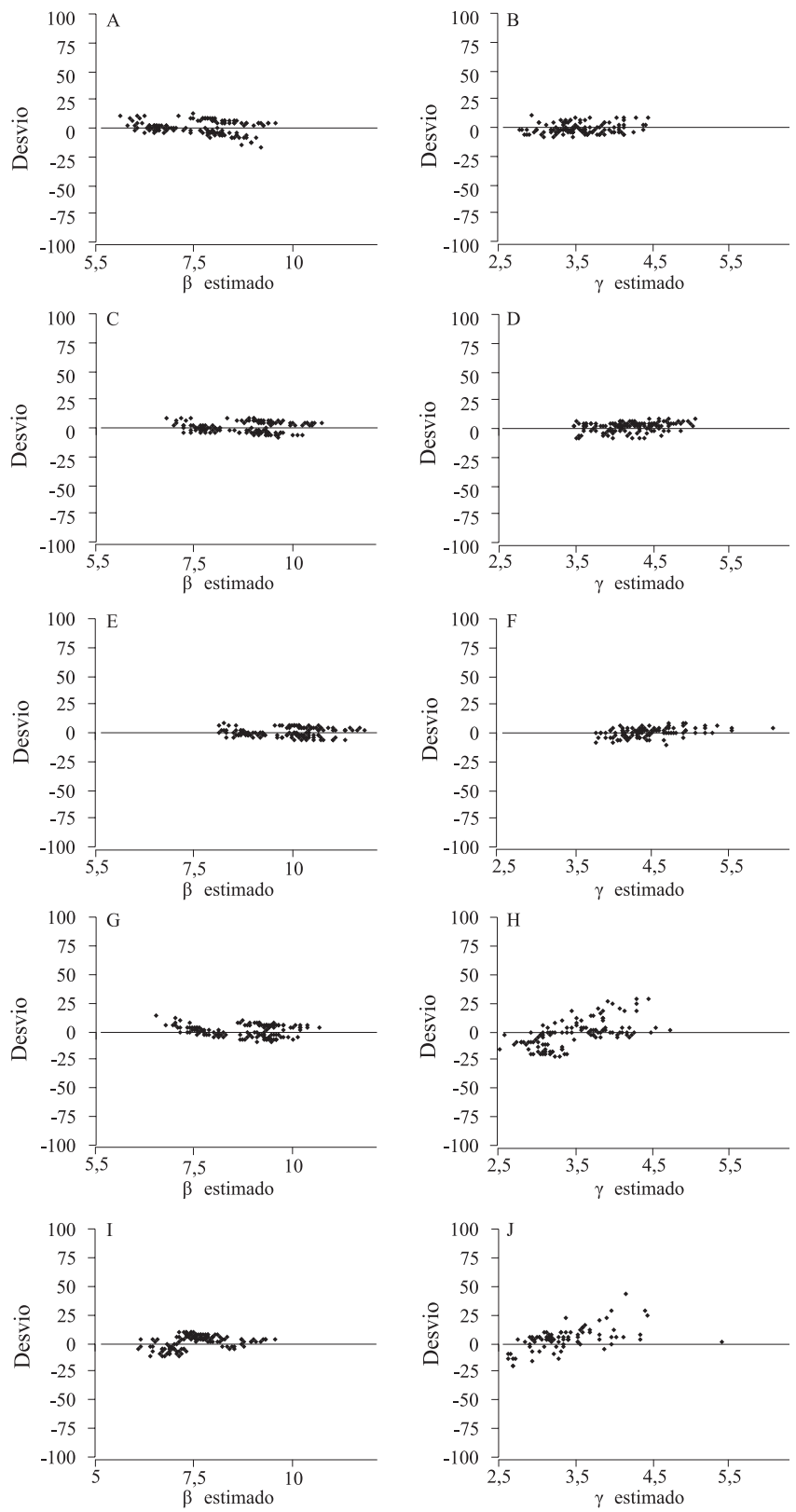

Figura 2. Valores estimados dos parâmetros $\beta \mathrm{e} \gamma$ da função Weibull e distribuição dos desvios para as amplitudes de classe 1,0 (A, B), 2,0 (C, D), 3,0 (E, F), 4,0 (G, H) e 5,0 (I, J) $\mathrm{cm}$. Desvio $=100(\beta$ recuperado $-\beta$ estimado $) / \beta$ estimado. 
Tabela 4. Média das diferenças (MD), média das diferenças percentuais (MDP) e raiz quadrada do erro médio (RQEM), com os valores de volume observado e de volumes calculados a partir das distribuições diamétricas estimadas pela função Weibull em cada idade (em meses), e para as distribuições prognosticadas pelo modelo de distribuição diamétrica ajustado.

\begin{tabular}{|c|c|c|c|c|c|c|c|c|c|c|c|c|c|c|c|c|c|c|c|}
\hline \multirow{2}{*}{$\begin{array}{l}\text { Amplitude de } \\
\text { classe }(\mathrm{cm})\end{array}$} & \multirow{2}{*}{$\begin{array}{l}\text { Centro de } \\
\text { classe }(\mathrm{cm})\end{array}$} & \multicolumn{6}{|c|}{ MD } & \multicolumn{6}{|c|}{ MDP } & \multicolumn{6}{|c|}{ RQEM } \\
\hline & & $40^{(1)}$ & $27-40^{(2)}$ & $50^{(1)}$ & $27-50^{(2)}$ & $58^{(1)}$ & $27-58^{(2)}$ & $40^{(1)}$ & $27-40^{(2)}$ & $50^{(1)}$ & $27-50^{(2)}$ & $58^{(1)}$ & $27-58^{(2)}$ & $40^{(1)} 2$ & $27-40^{(2)}$ & $50^{(1)}$ & $27-50^{(2)}$ & $58^{(1)}$ & $27-58^{(2)}$ \\
\hline 1,0 & 5,5 & $-0,38$ & $-0,31$ & $-0,47$ & $-0,27$ & $-0,53$ & $-0,49$ & $-88,23$ & $-80,24$ & $-88,30$ & $-82,31$ & $-89,07$ & $-85,14$ & 0,39 & 0,34 & 0,50 & 0,30 & 0,55 & 0,53 \\
\hline 1,0 & 6,5 & $-0,29$ & $-0,13$ & $-0,52$ & $-0,25$ & $-0,60$ & $-0,53$ & $-37,96$ & $-13,91$ & $-64,38$ & $-40,61$ & $-62,62$ & $-55,10$ & 0,36 & 0,27 & 0,58 & 0,33 & 0,67 & 0,64 \\
\hline 1,0 & 7,5 & 0,17 & 0,61 & $-0,41$ & 0,12 & $-0,46$ & $-0,25$ & 14,15 & 41,94 & $-18,04$ & 36,89 & $-16,32$ & 8,92 & 0,56 & 0,95 & 0,62 & 0,66 & 0,69 & 1,04 \\
\hline 1,0 & 8,5 & 1,54 & 2,16 & 0,59 & 1,39 & 0,64 & 1,09 & 43,87 & 61,55 & 27,45 & 88,75 & 29,59 & 54,10 & 1,87 & 2,46 & 1,02 & 1,72 & 1,17 & 2,01 \\
\hline 1,0 & 9,5 & 3,21 & 3,56 & 3,11 & 3,80 & 3,21 & 3,98 & 46,55 & 52,38 & 87,25 & 158,81 & 83,71 & 115,63 & 3,47 & 3,93 & 3,37 & 4,04 & 3,43 & 4,65 \\
\hline 1,0 & 10,5 & 2,37 & 1,85 & 5,00 & 5,44 & 5,48 & 6,49 & 18,68 & 15,38 & 73,68 & 115,72 & 81,09 & 100,76 & 3,18 & 3,06 & 5,19 & 5,63 & 5,67 & 7,00 \\
\hline 1,0 & 11,5 & $-6,08$ & $-7,51$ & 5,41 & 5,08 & 6,28 & 7,24 & $-22,36$ & $-28,03$ & 46,73 & 62,84 & 56,54 & 67,70 & 6,90 & 8,22 & 5,86 & 5,48 & 6,62 & 7,95 \\
\hline 1,0 & 12,5 & $-7,41$ & $-8,93$ & $-2,83$ & $-2,49$ & $-1,88$ & $-1,45$ & $-29,33$ & $-35,09$ & $-8,20$ & $-11,00$ & $-2,40$ & $-0,34$ & 7,96 & 9,69 & 5,19 & 4,21 & 5,77 & 5,84 \\
\hline 1,0 & 13,5 & 1,85 & 1,11 & $-13,28$ & $-11,96$ & $-13,29$ & $-13,85$ & 50,76 & 53,13 & $-35,22$ & $-46,80$ & $-33,88$ & $-35,87$ & 2,96 & 3,87 & 14,15 & 12,38 & 14,45 & 14,60 \\
\hline 1,0 & 14,5 & 2,96 & 2,94 & $-9,49$ & $-10,70$ & $-11,11$ & $-12,82$ & 275,14 & 297,32 & $-30,45$ & $-51,70$ & $-32,23$ & $-35,30$ & 3,15 & 3,34 & 10,53 & 11,63 & 12,28 & 15,51 \\
\hline 1,0 & 15,5 & 1,10 & 1,29 & 1,86 & $-2,83$ & $-1,12$ & $-3,60$ & 160,73 & 149,59 & 107,29 & 22,41 & 104,99 & 122,56 & 1,20 & 1,43 & 5,68 & 6,38 & 8,57 & 14,23 \\
\hline 1,0 & 16,5 & - & 0,59 & 4,51 & 0,04 & 4,17 & 1,63 & - & - & 284,10 & 98,07 & 312,89 & 323,29 & - & 0,68 & 4,94 & 2,67 & 5,20 & 7,88 \\
\hline 1,0 & 17,5 & - & 0,56 & 3,15 & 0,52 & 3,98 & 2,00 & - & - & 344,37 & 15,17 & 259,10 & 104,72 & - & 0,56 & 3,55 & 0,91 & 4,60 & 3,37 \\
\hline 1,0 & 18,5 & - & - & 1,59 & 0,58 & 2,48 & 1,07 & - & - & - & - & 340,40 & $-23,27$ & - & - & 1,85 & 0,64 & 3,02 & 1,61 \\
\hline 1,0 & 19,5 & - & - & 1,12 & 0,40 & 1,94 & 1,05 & - & - & - & - & - & - & - & - & 1,20 & 0,40 & 2,20 & 1,16 \\
\hline 1,0 & 20,5 & - & - & - & - & 1,18 & 0,75 & - & - & - & - & - & - & - & - & - & - & 1,21 & 0,75 \\
\hline 2,0 & 5,0 & $-0,19$ & $-0,18$ & $-0,25$ & $-0,24$ & $-0,29$ & $-0,34$ & $-71,60$ & $-68,36$ & $-80,62$ & $-79,12$ & $-80,75$ & $-85,25$ & 0,21 & 0,21 & 0,28 & 0,27 & 0,31 & 0,36 \\
\hline 2,0 & 7,0 & $-0,01$ & $-0,06$ & $-0,88$ & $-0,85$ & $-1,02$ & $-1,51$ & $-0,75$ & $-0,98$ & $-38,07$ & $-32,96$ & $-39,12$ & $-55,09$ & 0,39 & 0,60 & 0,95 & 1,16 & 1,08 & 1,71 \\
\hline 2,0 & 9,0 & 3,78 & 2,83 & 2,87 & 3,13 & 2,95 & 0,48 & 35,20 & 27,79 & 46,42 & 58,34 & 43,74 & 16,10 & 4,04 & 3,51 & 3,28 & 4,35 & 3,35 & 3,20 \\
\hline 2,0 & 11,0 & $-3,68$ & $-5,25$ & 9,46 & 10,18 & 10,77 & 6,15 & $-8,51$ & $-12,43$ & 49,52 & 56,20 & 57,48 & 38,06 & 5,02 & 6,32 & 9,73 & 11,28 & 10,98 & 8,71 \\
\hline 2,0 & 13,0 & $-4,93$ & $-2,40$ & $-14,36$ & $-13,96$ & $-13,23$ & $-12,82$ & $-12,37$ & $-2,73$ & $-22,47$ & $-21,84$ & $-19,73$ & $-18,98$ & 6,27 & 7,02 & 15,71 & 15,39 & 15,75 & 15,50 \\
\hline 2,0 & 15,0 & 4,62 & 7,54 & $-6,71$ & $-8,05$ & $-11,05$ & $-0,09$ & 351,57 & 611,78 & $-10,61$ & $-8,47$ & $-15,86$ & 17,10 & 4,84 & 8,13 & 10,62 & 18,16 & 15,64 & 21,75 \\
\hline 2,0 & 17,0 & 0,51 & 0,93 & 7,65 & 5,84 & 8,17 & 17,59 & - & - & 405,91 & 470,07 & 404,16 & 1160,49 & 0,52 & 1,10 & 8,02 & 8,82 & 8,64 & 21,77 \\
\hline 2,0 & 19,0 & - & - & 1,73 & 1,22 & 3,01 & 4,70 & - & - & 333,62 & $-62,65$ & 433,63 & 112,69 & - & - & 2,13 & 1,53 & 3,94 & 5,51 \\
\hline 2,0 & 21,0 & - & - & - & - & 1,12 & 1,14 & - & - & - & - & - & - & - & - & - & - & 1,17 & 1,28 \\
\hline 3,0 & 4,5 & $-0,07$ & $-0,10$ & $-0,16$ & $-0,15$ & $-0,18$ & $-0,22$ & $-31,10$ & $-50,13$ & $-68,84$ & $-64,86$ & $-68,80$ & $-76,33$ & 0,09 & 0,13 & 0,17 & 0,18 & 0,20 & 0,24 \\
\hline 3,0 & , & 1,48 & $-0,38$ & $-0,69$ & $-1,54$ & $-0,83$ & $-2,89$ & 26,88 & $-5,32$ & $-14,84$ & $-28,89$ & $-15,66$ & $-51,49$ & 1,64 & 1,50 & 0,85 & 2,29 & 1,00 & 3,37 \\
\hline 3,0 & 10,5 & $-3,63$ & $-7,83$ & 11,12 & 3,48 & 12,28 & $-2,67$ & $-7,45$ & $-16,42$ & 49,92 & 19,95 & 54,95 & $-5,90$ & 4,38 & 8,68 & 11,40 & 7,06 & 12,51 & 7,88 \\
\hline 3,0 & 13,5 & $-0,28$ & 12,50 & $-21,80$ & $-21,49$ & $-22,25$ & $-28,57$ & 1,40 & 39,32 & $-23,54$ & $-23,17$ & $-23,04$ & $-29,54$ & 3,20 & 15,12 & 22,29 & 22,47 & 22,96 & 29,42 \\
\hline 3,0 & 16,5 & 3,07 & 7,51 & 9,90 & 32,32 & 7,66 & 53,99 & 444,78 & 973,08 & 145,16 & 557,28 & 130,24 & 788,72 & 3,31 & 7,86 & 10,58 & 37,07 & 10,91 & 61,50 \\
\hline 3,0 & 19,5 & - & 0,63 & 2,25 & 5,91 & 4,21 & 25,92 & - & - & 423,86 & 254,03 & 572,58 & 1347,25 & - & 0,64 & 2,88 & 6,53 & 5,61 & 26,51 \\
\hline 3,0 & 22,5 & - & - & - & 0,87 & - & 2,13 & - & - & - & - & - & - & - & - & - & 0,87 & - & 2,56 \\
\hline 4,0 & 6 , & 0,68 & $-0,10$ & $-0,87$ & $-0,50$ & $-1,02$ & $-0,95$ & 34,92 & $-6,08$ & $-44,08$ & $-22,57$ & $-44,91$ & $-39,66$ & 0,71 & 0,71 & 0,90 & 1,04 & 1,04 & 1,33 \\
\hline 4,0 & 10,0 & $-3,82$ & $-3,55$ & 9,86 & 9,87 & 11,36 & 4,77 & $-7,82$ & $-6,70$ & 41,64 & 44,61 & 47,58 & 24,59 & 4,23 & 5,89 & 10,13 & 11,20 & 11,60 & 8,42 \\
\hline 4,0 & 14,0 & 1,36 & 7,29 & $-22,89$ & $-27,29$ & $-25,88$ & $-26,60$ & 5,33 & 24,32 & $-20,96$ & $-24,64$ & $-22,05$ & $-22,18$ & 3,37 & 11,42 & 23,36 & 30,31 & 26,33 & 30,55 \\
\hline 4,0 & 18,0 & 3,03 & 2,94 & 13,90 & 16,34 & 15,10 & 37,45 & - & - & 697,02 & 910,40 & 639,14 & 1844,89 & 3,35 & 3,62 & 14,09 & 17,88 & 15,29 & 39,23 \\
\hline 4,0 & 22,0 & - & - & 0,95 & 1,98 & 1,60 & 4,93 & - & - & - & - & - & - & - & - & 0,97 & 2,13 & 1,80 & 5,98 \\
\hline 5,0 & 7,5 & 0,74 & 6,99 & 1,46 & 8,92 & 1,55 & 7,26 & 6,06 & 67,57 & 19,17 & 121,30 & 18,21 & 90,51 & 0,90 & 7,28 & 1,56 & 9,38 & 1,64 & 7,97 \\
\hline 5,0 & 12,5 & $-6,04$ & $-31,87$ & $-8,99$ & $-37,94$ & $-9,04$ & $-34,47$ & $-7,19$ & $-36,84$ & $-8,67$ & $-35,94$ & $-8,41$ & $-31,49$ & 6,58 & 32,38 & 9,41 & 38,54 & 9,56 & 36,01 \\
\hline 5,0 & 17,5 & 7,53 & 17,11 & 8,61 & 15,57 & 7,49 & 18,16 & 742,17 & 1854,83 & 82,56 & 307,66 & 72,28 & 365,62 & 7,78 & 18,20 & 8,92 & 26,61 & 8,83 & 37,49 \\
\hline 5,0 & 22,5 & - & 1,64 & 2,32 & 5,24 & 4,99 & 9,56 & - & - & - & - & - & - & - & 1,76 & 2,55 & 5,85 & 5,76 & 10,59 \\
\hline$\underline{5,0}$ & 27,5 & - & - & - & - & - & 1,41 & - & - & - & - & - & - & - & - & - & - & - & 1,42 \\
\hline
\end{tabular}

${ }^{(1)}$ Resultados em que foi considerada a distribuição estimada pela função Weibull, para a respectiva idade em meses; ${ }^{(2)}$ Resultados em que foi considerada a distribuição diamétrica recuperada pelo modelo de distribuição diamétrica, com idade inicial de 27 meses e projeção para a respectiva idade em meses.

e prognosticadas, verificou-se que as diferenças entre os valores seguiram uma tendência bem definida entre diferentes idades, ou seja, o modelo de distribuição diamétrica, ao prognosticar as frequências de árvores para cada classe em idades futuras, apresentou erros semelhantes aos gerados pelas estimativas da função Weibull em cada idade, o que está de acordo com os resultados obtidos no ajuste das equações que compõem o modelo apresentado.

No entanto, ao se comparar diferentes amplitudes, verificou-se que a tendência mencionada anteriormente não se manteve. Para as amplitudes de 3,0, 4,0 e $5,0 \mathrm{~cm}$, houve maior discrepância entre os valores das estatísticas, o que não ocorreu para as amplitudes de 
1,0 e $2,0 \mathrm{~cm}$. Tal inconsistência pode estar associada à falta de tendência definida para os parâmetros da função Weibull, já que a forma da distribuição foi drasticamente alterada com a quantidade de classes em cada idade (Figura 1).

Observou-se também que maiores amplitudes de classe ocasionaram perdas de informações acerca do comportamento da distribuição diamétrica e resultaram em inconsistências nos cálculos de projeção. Assim, a definição da amplitude de classe interferiu na qualidade da prognose e, consequentemente, na precisão das estimativas atuais e futuras da produção volumétrica total e por classe de diâmetro.

\section{Conclusões}

1. A função densidade de probabilidade Weibull, independentemente da amplitude de classe considerada, é adequada para descrever a distribuição diamétrica de povoamentos equiâneos de eucalipto em diferentes idades.

2. A amplitude de classe afeta a qualidade da prognose das frequências de indivíduos por classe de diâmetro.

\section{Referências}

ABREU, E.C.R.; SCOLFORO, J.R.S.; OLIVEIRA, A.D. de; MELLO, J.M. de; KANEGAE JÚNIOR, H. Modelagem para prognose precoce do volume por classe diamétrica para Eucalyptus grandis. Scientia Forestalis, n.61, p.82-102, 2002.

ARCE, J.E. Modelagem da estrutura de florestas clonais de Populus deltoides Marsh através de distribuições diamétricas probabilísticas. Ciência Florestal, v.14, p.149-164, 2004.

AYALA, D.M.; NÁVAR-CHÁIDEZ, J. Ajuste y predicción de la distribución Weibull a las estructuras diamétricas de plantaciones de pino de Durango, México. Madera y Bosques, v.8, p.61-72, 2002.

BAILEY, R.L.; DELL, T.R. Quantifying diameter distributions with the Weibull function. Forest Science, v.19, p.97-104, 1973.

CAMPOS, J.C.C.; LEITE, H.G. Mensuração florestal: perguntas e respostas. 3.ed. Viçosa: UFV, 2009. 548p.

CAO, Q.V. Predicting parameters of a Weibull function for modeling diameter distribution. Forest Science v.50, p.682-685, 2004.

CATALUNHA, M.J.; SEDIYAMA, G.C.; LEAL, B.G.; SOARES, C.P.B.; RIBEIRO, A. Aplicação de cinco funções densidade de probabilidade a séries de precipitação pluvial no Estado de Minas
Gerais. Revista Brasileira de Agrometeorologia, v.10, p.153-162, 2002.

CUNHA, U.S. da; MACHADO, S. do A.; FIGUEIREDO FILHO, A.; SANQUETTA, C.R. Predição da estrutura diamétrica de espécies comerciais de terra firme da Amazônia por meio de matriz de transição. Ciência Florestal, v.12, p.109-122, 2002.

EISFELD, R. de L.; SANQUETTA, C.R.; ARCE, J.E.; MAESTRI, R.; WEBER, K.S. Modelagem do crescimento e da produção de Pinus taeda L. por meio de função probabilística. Floresta, v.35, p.317-328, 2005.

GOVE, J.H.; FAIRWEATHER, S.E. Maximum-likelihood estimation of Weibull function parameters using a general interactive optimizer and grouped data. Forest Ecology and Management, v.28, p.61-69, 1989.

LEITE, H.G.; NOGUEIRA, G.S.; CAMPOS, J.C.C.; TAKIZAWA, F.H.; RODRIGUES, F.L. Um modelo de distribuição diamétrica para povoamentos de Tectona grandis submetidos a desbaste. Revista Árvore, v.30, p.89-98, 2006.

MABVURIRA, D.; MALTAMO, M.; KANGAS, A. Predicting and calibrating diameter distributions of Eucalyptus grandis (Hill) Maiden plantations in Zimbabwe. New Forests, v.23, p.207-223, 2002.

MURPHY, P.A.; STERNITZKE, H.S. Growth and yield estimation for loblolly pine in the West Gulf. New Orleans: US Department of Agriculture, 1979. 8p. (Research paper, SO-154).

NÁVAR-CHÁIDEZ, J.J.; CONTRERAS-AVIÑA, J. Ajuste de la distribución Weibull a las estructuras diamétricas de rodales irregulares de pino em Durango, México. Agrociencia, v.34, p.353-362, 2000.

NOGUEIRA, G.S. Modelagem do crescimento e da produção de povoamentos de Eucalyptus sp. e de Tectona grandis submetidos a desbaste. 2003. 132p. Tese (Doutorado) - Universidade Federal de Viçosa, Viçosa.

NOGUEIRA, G.S.; LEITE, H.G.; CAMPOS, J.C.C.; CARVALHO, A.F.; SOUZA, A.L. de. Modelo de distribuição diamétrica para povoamentos de Eucalyptus sp. submetidos a desbaste. Revista Árvore, v.29, p.579-589, 2005.

SCOLFORO, J.R.S.; THIERSCHI, A. Estimativas e testes da distribuição de freqüência diamétrica para Eucalyptus camaldulensis, através da distribuição $\mathrm{Sb}$, por diferentes métodos de ajuste. Scientia Forestalis, n.54, p.93-106, 1998.

SOARES, T.S. Avaliação de modelos de distribuição diamétrica em povoamentos de eucalipto não desbastados. 2006. 69p. Tese (Doutorado) - Universidade Federal de Viçosa, Viçosa.

UMAÑA, C.L.A.; ALENCAR, J.C. Distribuições diamétricas da floresta tropical úmida em uma área no Município de Itacoatiara AM. Acta Amazonica, v.28, p.167-190, 1998.

Recebido em 5 de maio de 2010 e aprovado em 13 de setembro de 2010 